\title{
Consistency and Correction of Line-Drawings, Obtained by Projections of Piecewise Planar Objects
}

\author{
Anders Heyden \\ Dept of Mathematics, Lund Institute of Technology, P.O. Box 118, S-221 00 Lund, \\ Sweden
}

\begin{abstract}
This paper deals with line-drawings, obtained from images of piecewise planar objects after edge detection. Such images are used e.g. for navigation and recognition. In order to be a possible image of a three dimensional piecewise planar object, it has to obey some projective conditions. Criteria for a line-drawing to be correct is given in this paper, along with methods to find possible interpretations. If there are many objects in the scene there may be occlusions between objects. There may also be occlusions between different parts of the same object. A method that can handle such occlusions is presented.
\end{abstract}

Keywords. Line-drawings, projection, depth, shape, occlusion.

\section{Introduction}

A central problem in scene analysis is the reconstruction of $3 \mathrm{D}$-objects from $2 \mathrm{D}$ images, obtained by projections. Two important classes of objects are polyhedral and origami ('papermade') ones, where the difference is that in the latter case the objects need not be solid, but may partly consist of laminas. Projective images of such objects will, after edge detection, be called Line-drawings. Conditions are imposed on the line-drawing by the projective image formation process. There exist line-drawings which are impossible as images of truly three dimensional objects. One well-known example is the Reuterswärd-Penrose tribar, see [Penrose].

Another conceivable application for the correction methods is in computer aided design (CAD), where a careless line drawing may serve as input, and a projectively correct line drawing is obtained as output.

In this paper we will concentrate on different criteria for correctness of a linedrawing and introduce a new criteria which is easier to handle than earlier ones. We will also discuss the degree of freedom in a line-drawing. Finally we will show how to handle occlusions and make a comparision with Sugiharas method, see [Sugihara 86].

\section{Depth and Shape}

In this chapter we will present some basic properties of the concepts of deptli and shape. For more detailed treatments, see [Sparr 91], [Sparr 92]. We start with the definition of shape. 
Definition 1. A configuration is an ordered set of points, $X^{n}=\left(X^{1}, \ldots, X^{n}\right)$, in 3 -space. Let $x^{i}$ denote their coordinates in some basis, $i=1, \ldots, n$. Then the shape of $\mathcal{X}$ is defined as the linear space

$$
s(\mathcal{X})=\left\{\xi \mid \sum_{i=1}^{n} \xi_{i} x^{i}=0, \sum_{i=1}^{n} \xi_{i}=0\right\}=\mathcal{N}\left[\begin{array}{cccc}
1 & 1 & \ldots & 1 \\
x^{1} & x^{2} & \ldots & x^{n}
\end{array}\right] .
$$

An important property of $s\left(x^{\prime}\right)$ is that it is independent of the coordinate representation of the points, or (equivalently) that it is unchanged under affine coordinate transformations. A perspective transformation (or perspectivity) with center $Z$ and image plane $\pi, Z \notin \pi$, is a mapping with the property that every point on a line through $Z$ is mapped onto the intersection of the line with $\pi$. A projectivity is a composition of perspectivities.

The importance of these concepts is illustrated in the following theorems and definitions.

Theorem 2. If $\mathcal{X}$ and $\mathcal{Y}$ are planar configurations, then the following statements are equivalent:

(1) There exists a perspectivity $P$, such that $P(\mathcal{X})$ and $\mathcal{Y}$ have equal shape, and $\mathcal{X}$ has depth a with respect to $P(\mathcal{X})$

(2) $\operatorname{diag}(\alpha) s\left(\mathcal{X}^{\prime}\right)=s(\mathcal{Y})$.

Proof. See [Sparr 92].

This theorem says that whenever an $\mathcal{X}$-configuration, with a given shape $s(\mathcal{X})$, can be mapped by a perspectivity onto a $\mathcal{Y}$-configuration, with a given shape $s(\mathcal{Y})$, that mapping must have the deptlh $\alpha$ given by the theorem, independently of $Z$ and $\pi$.

We will now consider point configurations defined by the vertices of piecewise planar objects. Each of the $f$ polygonal faces of the object contributes by its vertices with a planar subconfiguration, $\mathcal{X}_{i}=\left(X_{i}^{1}, \ldots, X_{i}^{n i}\right), \quad i=1, \ldots, f$. Thus the whole configuration can be considered as an ordered set of such sub-configurations, that is as a configuration of configurations, which will be called a composite configuration and will be denoted by $(\mathcal{X})=\left(\mathcal{X}_{1}, \ldots, \mathcal{X}_{f}\right)$.

In the sequel, these concepts will be used to analyze piecewise planar objects and their projective images. Such an object consists of planar polygonal regions, also called faces. For each such polygon the vertices form a planar subconfiguration. The object will be identified with the composite configuration built up by these subconfigurations. Concerning the image of a piecewise planar object, it is assumed that for each pair of adjacent faces in the object their intersection give rise to a linear arc in the image plane. These arcs together form what is called a line-drawing, consisting of a number of coplanar polygonal regions.

Below we deal with the problem of reconstruction of the (class of) objects that are possible to project on to a given line-drawing. The objects are assumed to be non-transparent. Since the line-drawing only contains information about the visible parts of the object, the word 'object' will be used synonymously with 'visible parts of the object'. We will also consider the situation of a scene consisting of several objects, where occlusions cause severe complications. 
Definition 3. If $\mathcal{X}$ is a point configuration, by an $S$-matrix for $\mathcal{X}$ is meant a matrix $S^{\mathcal{X}}$ whose columns form a basis for $s(\mathcal{X})$. If $(\mathcal{X})=\left(\mathcal{X}_{1}, \ldots, \mathcal{X}_{f}\right)$ is a composite configuration, by an $S$-matrix for $(\mathcal{X})$ is meant a matrix $S^{(\mathcal{X})}=\left[S_{1} \ldots S_{f}\right]$ such that, for $i=1, \ldots, f$ : each point of $\mathcal{X}$ corresponds to a row of $S^{\left(x^{\prime}\right)}, S_{i}$ corresponds to the face $X_{i}, S_{i}$ has zeros on all rows corresponding to points not in $X_{i}$, and $S_{i}$ can be obtained from $\left[S^{X_{i}} 0\right]^{T}$ by a permutation of the rows. By the composite shape space $s\left(\left(X^{\prime}\right)\right)$ is meant the column space of $S^{(\mathcal{X})}$.

Note that the $S$-matrix is not uniquely defined. Also note that the composite configuration $(X)$ contains more structure than the configuration $\mathcal{X}$, because in $(\mathcal{X})$ the points are ordered in subconfigurations. From the definition follows that there are more columns in $S^{\mathcal{X}}$ than in $S^{(\mathcal{X})}$, because every column in $S^{(\mathcal{X})}$ belongs to $s(\mathcal{X})$, but together they don't necessarily span $s(\mathcal{X})$, hence $s((\mathcal{X})) \subseteq s(\mathcal{X})$. The $S$-matrices tell us a lot about the original configuration, which the next theorem shows.

Theorem 4. Let $(\mathcal{X})$ be a composite configuration and let $(\mathcal{Y})$ be its image under a projective transformation. with known point correspondences. Then to every choice of $S^{(\mathcal{X})}$ there exists an $S$-matrix $S^{(\mathcal{Y})}$ with the same distribution of zeroes as $S^{(\mathcal{X})}$. For these $S$-matrices hold for some a and $c$,

$$
\operatorname{diag}(\alpha) S^{(\mathcal{X})}=S^{(\mathcal{y})} \operatorname{diag}(c) \Leftrightarrow \operatorname{diag}(\alpha) s((\mathcal{X}))=s((\mathcal{Y})) .
$$

Proof. See [Sparr 92].

Given a planar composite configuration $(\mathcal{Y})$, we say that a composite configuration (possibly three-dimensional), $(\mathcal{X})$, is a pre-image of $(\mathcal{Y})$ if there is a projective transformation taking the points in $(\mathcal{X})$ to the points in $(\mathcal{Y})$. According to the previous theorem $(\mathcal{X})$ is a preimage of $(\mathcal{Y})$ if and only if there exists $S^{(\mathcal{X})}$ and $S^{(\mathcal{Y})}$ fulfilling (2) for some $\alpha$ and $c$. The next theorem tells us, for a given planar composite configuration $(\mathcal{Y})$, which depths that can occur for a perspective transformation of a composite configuration with image $(\mathcal{Y})$.

Theorem 5. Given a planar composite configuration, $(\mathcal{Y})$, and an $S$-matrix, $S^{(\mathcal{Y})}$. Then the set of possible depth vectors $\alpha=\left[\alpha_{1} \ldots \alpha_{n}\right]^{T}$ for $\mathcal{Y}$ with respect to some preimage is given by the solution to

$$
\beta^{T} S^{(\mathcal{Y})}=0, \quad \beta=\left[\alpha_{1}^{-1} \ldots \alpha_{n}^{-1}\right]^{T},
$$

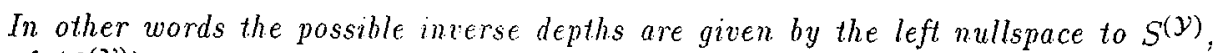
$\mathcal{N}_{L}\left(S^{(\mathcal{Y})}\right)$.

Proof. According to Theorem $4 \operatorname{diag}(\alpha) S^{\left(x^{x}\right)}=S^{(\mathcal{y})}$ where $S^{(x)}$ is an $S$-matrix of some preimage. The fact that $S^{(x)}$ is an $S$-matrix means that each of its column sums vanishes, which is the same as $\epsilon^{T} S^{(\mathcal{X})}=0 \Rightarrow e^{T} \operatorname{diag}(\beta) S^{(\mathcal{Y})}=0 \Rightarrow \beta^{T} S^{(\mathcal{Y})}=$ 0 where $e=\left[\begin{array}{llll}1 & 1 & \ldots\end{array}\right]^{T}$.

\section{Criteria for Correctness}

In this section we first discuss what is meant by a correct line-drawing and how to use the $S$-matrix to determine if a composite configuration is correct. 
Next we have to define what to mean by a possible/impossible line-drawing of a piecewise planar object. As is discussed above such a line-drawing is obtained by projection from a piecewise planar composite configuration where every pair of adjacent faces intersect in a line. A human person would in general interpret the lines in a line-drawing as caused by such intersections of planes in the scene. Then a linedrawing is correct if there exists a preimage where no pair of planes corresponding to opposite faces of a line coincide. This criterion will be called truly possible or truly correct. Another way to say this is that every pair of adjacent faces in the line-drawing corresponds to non-parallel planes in the preimage. Sugihara has used a slightly stronger condition requiring no planes at all in the preimage to be parallel, see [Sugihara 86]. This criteria will be called strictly possible or strictly correct. These definitions of correctness have an intuitively good interpretation but they are hard to work with. Therefore we give a third definition of correctness.

Definition 6. A planar composite configuration, $(\mathcal{Y})$, will be called weakly impossible or weakly incorrect if for some connected collection of faces $(\mathcal{A})=$ $\left(\mathcal{Y}_{i_{1}}, \ldots, \mathcal{Y}_{i_{k}}\right), k \geq 2$, holds

$$
P:(\mathcal{X}) \longrightarrow(\mathcal{A}), \quad P \text { a projectivity } \Longrightarrow(\mathcal{X}) \text { is a planar configuration. }
$$

This is a slightly weaker condition than the one used by Sugihara. The relations between them are

$(\mathcal{Y})$ is strictly correct $\Rightarrow(\mathcal{Y})$ is truly correct $\Rightarrow(\mathcal{Y})$ is weakly correct

Proof. If $(\mathcal{Y})$ is weakly impossible then there exists some connected collection, $(\mathcal{A})$, of faces that are coplanar in every preimage of $(\mathcal{A})$. Thus $(\mathcal{Y})$ is truly impossible, because there exist adjacent faces that are coplanar in every preimage of $(\mathcal{Y})$. If $(\mathcal{Y})$ is truly impossible then there exists a pair of adjacent faces that are coplanar in every preimage of $(\mathcal{Y})$ and thus $(\mathcal{Y})$ is strictly impossible.

Theorem 7. $(\mathcal{Y})$ is a weakly impossible planar composite configuration if and only if

$$
\operatorname{rank} S^{(\mathcal{A})} \geq|V(\mathcal{A})|-3
$$

for some collection of faces $(\mathcal{A})$ of $(\mathcal{Y})$, where $|V(\mathcal{A})|$ is the number of points in $\mathcal{A}$.

Proof. For any preimage $(\mathcal{X})$ of the planar composite configuration $(\mathcal{A})$ holds

$$
\operatorname{diag}(\alpha) s((\mathcal{X}))=s((\mathcal{A}))
$$

or equivalently,

$$
s((\mathcal{X}))=\operatorname{diag}(\alpha)^{-1} s((\mathcal{A}))
$$

Hence (4) holds for $(\mathcal{A})$ if and only if it holds for $(\mathcal{X})$, which in turn, is the same as that $(\mathcal{X})$ is planar.

Theorem 7 shows that it is possible to use the $S$-matrix $S^{(\mathcal{Y})}$ to determine if the planar composite configuration $(\mathcal{Y})$ is weakly possible.

Definition 8. We say that $(\mathcal{Y})$ obeys the rank condition if rank $S^{(\mathcal{A})} \leq|V(\mathcal{A})|-4$ for every collection of faces, $(\mathcal{A})$, of $(\mathcal{Y})$. Here again $|V(\mathcal{A})|$ denotes the number of points in $\mathcal{A}$. 


\section{The Degree of Freedom}

We begin with the following definition.

Definition 9. The degree of freedom of a planar composite configuration is the maximal number of vertices in a preimage that can be independently specified. This is the same as the maximal number of depths that can be independently prescribed.

We say that two preimages differ if their respective depths are not proportional as vectors in $\mathbb{R}^{n}$. Further we say that the number of different preimages is the same as the dimension of the linear space of possible depths. This can be written in the language of matroids (see [White 86]) as follows. Let $(\mathcal{Y})$ be a planar composite configuration. From Theorem 5 we know that $\alpha^{T} S^{(\mathcal{Y})}=0$ gives the possible depths. To specify one point in $\mathbb{R}^{3}$, i.e. to give it a prescribed depth value, corresponds to imposing an equation of the form $\alpha^{T} e_{i}=d_{i}$, where $e_{i}$ is the $i$ :th unit vector and $d_{i}$ is the prescribed depth. We also observe that rank $S^{(y)}=|V|-k$ means that $k$ depths can be specified (at most), where $|V|$ means the number of points in $\mathcal{Y}$. Then we get the following theorem.

Theorem 10. The function $p_{V}: 2^{V} \rightarrow \mathbb{R}$ defined by

$$
\rho_{V}(X)=\operatorname{rank}\left(\left[S^{(\mathcal{Y})} \mid I_{X}\right]\right)-\operatorname{rank}\left(S^{(\mathcal{Y})}\right)
$$

is the rank function of a matroid. Here $\left[S^{(\mathcal{Y})} \mid I_{X}\right]$ is a block matrix with the second block $I_{X}$ composed by columns $e_{i}$, unit vectors, with $i$ corresponding to points in $X$. $\rho_{V}(X)$ is the maximal number of the depths in $X$ that can be specified independently.

Proof. See [Heyden 93b].

Given a subset $A$ of vertices, we call $\rho_{V}(A)$ the degree of freedom of $A$. Further $A$ is said to be independent if $\rho_{V}(A)=|A|$ and dependent otherwise. A maximal independent subset is called a base. One property of matroids is that every base has the same cardinality, that is if $\rho_{V}(A)=|A|$ and $\rho_{V}(B)=|B|$ then $|A|=|B|$. Further $\rho_{V}(V)=\rho_{V}(B)$ for any base $B$. This means that the degree of freedom is $\rho_{V}(V)$. We restate this as a theorem

Theorem 11. The degree of freedom in a planar composite configuration, $(\mathcal{Y})$, is $\rho_{V}(V)=|V|-\operatorname{rank}\left(S^{(\mathcal{Y})}\right)$.

This theorem can of course be proved by standard linear algebra, but to describe the degree of freedom for every subset of vertices or faces the matroid formulation is needed.

A base in the matroid can be constructed from one empty set $B$ by going through all vertices, $v$, adding them to $B$ if $B \bigcup\{v\}$ is independent, dropping them otherwise. Eventually $\rho_{V}(B)=\rho_{V}(V)$ and we get a base. This is called the 'greedy algorithm'.

In the sequel the term correct will always mean weakly correct.

\section{$5 \quad$ Objects with Occlusions}

So far we have not considered occluded objects, that is objects that in the image seem to be partly hidden by another object or by part of itself. In order to determine 
the correctness of such an image we have to allow that each point where two objects meet in the image corresponds to two points in the preimage, and the point on the occluded part is more distant from the camera than the point on the occluding part. We have to impose some restriction on the class of objects appearing in the scene. One method is to assume that at most three faces meet at a common point. Then all occlusions appear at 'T-crossings', where the occluding part is the top bar of the ' $T$ ' and the occluded part is the vertical line in the ' $T$ '. This was for instance done in [Sugihara 86], and will be done below.

Given a planar composite configuration $(\mathcal{Y})$. As stated before, where occlusion occurs, we have to introduce two points in the preimage, $\left(\mathcal{X}^{\prime}\right)$, instead of one. Then an $S$-matrix of $(\mathcal{Y}), S^{(\mathcal{Y})}$, is formed as usual, with some points coinciding. In this case it is not sufficient that $(\mathcal{Y})$ fulfils the rank condition in order to be correct. We must also impose that the depth value for the occluding point is less than the depth value for the occluded point in the preimage at every occlusion. Thus we need to know the depths for the different points in the image. The possible depth values in a preimage are given by Theorem 5 .

There are two different ways to cope with this problem. We have to solve a system of linear equations $\beta^{T} S^{(\mathcal{Y})}=0$ where $\beta=\left[\alpha_{1}^{-1}, \ldots, \alpha_{n}^{-1}\right]$ are the inverse depths, under some set of inequality constraints $\alpha_{i}<\alpha_{j}$. This gives a set of inverse inequalities in the inverse depths $\beta_{i}>\beta_{j}$. For a physical imaging process, it is assumed that the depths are greater than zero, because otherwise some point in the preimage is behind the camera. In our case, we have no problem with negative depths. Since $e=\left[\begin{array}{lll}1 & \ldots & 1\end{array}\right]^{T}$ is a vector of possible inverse depths, once we have a vector of inverse depths satisfying the inequalities above, by addition of $e$ and linearity we get an inverse depth vector with positive inverse depths satisfying the inequalities too. Observe that the inverse depths, but not the depths, form a linear space, the left nullspace of $S^{(\mathcal{Y})}$.

One way to compute $\beta$ is to solve the whole system of equations and inequalities at the same time. This can be done by linear programming. The solution is obtained by finding a basic feasible solution to the LP problem. This method was proposed by Sugihara in [Sugihara 86]. One drawback of the method is that the time complexity is very large.

Another way is to solve the system of equations first by finding a base of the left nullspace to the $S$-matrix (the number of vectors given depend on the degree of freedom of the image, see Section 4). Then we can discard the vectors lying in the space spanned by $e, x$ and $y$, because these vectors are not interesting in the lift of the image. Then we have to detect if some linear combination of the remaining vectors fulfil the inequality constraints. This is a standard problem of finding a point in a convex set defined by linear inequalities, and efficient methods exist (see [Schrijver]), based on LP-techniques. The method has a much lower degree of time complexity if the number of vectors obtained in the first step is small compared to the number of points in the image. Given $\beta^{1}, \beta^{2}, \ldots, \beta^{n}$, solutions to $\beta_{\tilde{\beta_{n}}}^{T} S^{(\mathcal{Y})}=0$, we want to find some linear combination $\tilde{\beta}=\sum_{i=1}^{n} \lambda_{i} \beta^{i}$ such that $\tilde{\beta}_{j}>\tilde{\beta}_{k}$ for some indices $j, k$. This means

$$
\left(\sum_{i=1}^{n} \lambda_{i} \beta^{i}\right)_{j}>\left(\sum_{i=1}^{n} \lambda_{i} \beta^{i}\right)_{k} \Longleftrightarrow \sum_{i=1}^{n}\left(\left(\lambda_{i} \beta^{i}\right)_{j}-\left(\lambda_{i} \beta^{i}\right)_{k}\right)>0 .
$$


Introducing $\left(\hat{\beta}^{i}\right)_{k}=\left(\beta^{i}\right)_{j}-\left(\beta^{i}\right)_{k}$ for $i=1, \ldots, n$ gives $\sum_{i=1}^{n}\left(\lambda_{i} \hat{\beta}^{i}\right)_{k}>0$. Define $M=\left\{\lambda \in \mathbb{R}^{n} \mid \lambda \cdot(\hat{\beta})_{k}>0\right\}$. This is a convex set (possibly empty) and if we find a point in $M$ we also have a solution to the problem above. The set of possible depths is thus given uniquely by the extremal points of $M$. If we require all components in the inverse depth vector to be positive and to have component sums one the extremal points are unique.

Example 1. One interesting example is the following planar composite configuration, see Figure 1. Here we have four inequalities involving the inverse depths $\beta_{10}>$
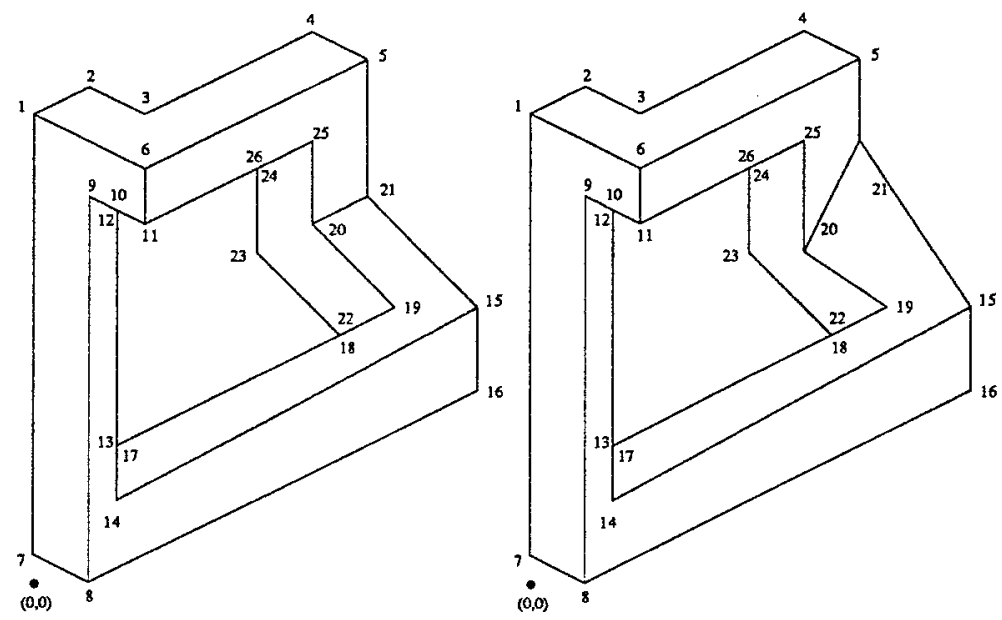

Fig. 1. Incorrect and correct composite configurations

$\beta_{12}, \beta_{13}>\beta_{17}, \beta_{18}>\beta_{22}, \beta_{26}>\beta_{24}$. The figure on the left has a base for the inverse depths at the points $10,12,13,17,18,22,24$ and 26 consisting of $e, x, y$ and $[-0.1132,0.0494,-0,071,-1.077,0.0815,-0.0695,-0.3170,-0.1661]$. Note that the minus signs do not cause any trouble according to the comment above. Positive depths can be obtained by addition of $e$. It can be seen that this vector does not fulfil the inequalities above (nor does minus this vector). Thus the first picture is incorrect.

The corresponding inverse depths for the figure on the right are $[0.2402,0.1649$, $0.0068,-0.1119,0.0886,-0.0894,-0.2920,0.0640]$. It can be seen that this vector fulfils the inequalities above. Thus the second picture is correct.

\section{Overcoming Superstrictness}

One problem that occurs when a planar composite configuration $(\mathcal{Y})$ is analyzed is that the $S$-matrix, $S^{(\mathcal{Y})}$, may not obey the rank condition exactly. When noise is affecting the image, every linear dependency between the columns in the $S$-matrix, 
which is necessary in order to fulfil the rank condition, is likely to be destroyed. When an $S$-matrix does not fulfil the rank condition but the submatrices built up by the subsets of columns that do not obey the rank condition has singular values that are small, it would be desirable to correct the $S$-matrix. This means finding a new $S$-matrix which is close to the previous one, in some sense, and obeys the rank condition.

If we want to correct the configuration we have to decrease the rank of these submatrices, which is the same as making some linear combinations of the columns vanish. These linear combinations can be found by a singular value decomposition. This kind of linear dependencies is called a second order syzygy. See [Heyden 93a] or [Persson 91] for different correction methods based on this observation and a model of how noise affect the $S$-matrix. The first order syzygies are the vectors in $S^{X_{i}}$, for each face $X_{i}$, with all components except four equal to zero, because they are minimal linear dependencies of the inverse depths of the points in the preimage.

Unfortunately this process does not stop at this second stage. There can be linear dependencies among the second order syzygies, called third order syzigies and so on.

It is an open problem to find all higher order syzygies to a composite configuration.

\section{A Comparison with Sugiharas Method}

In this section we will make same comments of how our method is related to that Sugihara has presented in [Sugilhara 86]. There are some similarities as well as some differences.

Sugihara starts with a consistently labelled line-drawing where each line in the image is labelled as convex, concave or occluding. This step is not needed in our method. We need the composite configuration and knowledge of where occlusion occur in order to build up the $S$-matrix and the inequality constraints. The composite configuration contains information about which points must by coplanar in every preimage. We use the convention (also used by Sugihara) that every ' $T$ '-crossing of lines represents an occlusion. We can look at the next step in the algorithm as telling us if this initial interpretation of the image is consistent with the laws of projective geometry.

Sugihara uses a different method to obtain the equations corresponding to Theorem 5 , but the equations are the same. The next step is to overcome superstrictness. Sugihara solves this by picking out points in the image until a substructure that has a preimage remains. Then he checks if the deleted points are close to lie in the planes in the preimage where they are supposed to be. One disadvantage of this approach is that all points in the image are not used in the same way. We have used a different approach and have tried to correct the $S$-matrix, so that all subsets of the columns obeys the rank condition. In order to do this we have to find all syzygies of order two and higher. This is a difficult problem and is yet not solved completely. Our solution holds for the common situation of images with only second order syzygies. 
Then Sugihara solves the set of equations given above by the algorithm used for finding a feasible starting point in a linear programming problem with these equations as restrictions. This is a rather complex algorithm, having both linear equations and inequalities. We have fewer unknown variables because the surface variables are not present and we have used another approach to solve the problem. First we find all solutions to the system of equations (this is rather easy) and then try to find if there are some linear combinations obeying the inequality constraints. This is a standard algorithm for finding a point in a convex set after some calculations.

The last step is to correct the image if possible. Sugihara just checks if the removed points obey the inequality constraints and if they nearly obeys the equality constraints. In our approach the $S$-matrix is already modified, and we can find new points consistent with this modified $S$-matrix see [Heyden 93a].

\section{Conclusions}

In this paper we have presented criteria for the correctness of an image of a piecewise planar object. The criterion weak correctness, involving the $S$-matrix, seems to be easier to work with than previous approaches. Further the degree of freedom in an image have been examined by matroid theory. An algorithm for dealing with images where occlusion occurs are given. The results of experiments are intuitively reasonable, as is illustrated in examples. The methods are able to generate interpretations of line-drawings in many, but not all, cases. For a complete solution, further work on finding all higher order syzygies of the S-matrix lias to be done.

\section{References}

[Heyden 93a] Heyden, A., Methods for Correction of Images of Origami/Polyhedral Objects, Proceedings of The 8 th Scandinavian Conference on Image Analysis, Tromsø, Norway, 1993.

[Heyden 93b] Heyden, A., On the Consistency of Line-Drawings, Obtained by Projections of Piecewise Planar Objects, CODEN:LUFTD2/TFMA-3001/5001-SE, Lund, Sweden, 1993.

[Penrose] Penrose R. On the Cohomology of Impossible Figures, Structural Topology, nr 17, 1991, pp. 11-16.

[Persson 91] Persson, A., A Method for Correction of Images of Origami/Polyhedral Objects, Proc. Symposium on Image Analysis, SSAB, Uppsala, Sweden, 1992, pp. 93-96.

[Schrijver] Schrijver, A., Theory for Linear and Integer Programming, John Wiley \& sons, Chichester, New York, Brisbane, Toronto, Singapore, 1986.

[Sparr 91] Sparr, G., Projective Invariants for Affine Shapes of Point Configurations, ESPRIT/DARPA Invariants Workshop, Reykjavik, Iceland, 1991.

[Sparr 92] Sparr, G., Depth-Computations from Polyhedral Images, ECCV'92, Lecture notes in Computer Science, Vol 588. Ed. G. Sandini, Springer-Verlag 1992, pp. 378-386. Also in Image and Vision Computing, Vol 10.1992, pp. 683-688.

[Sugihara 86] Sugihara, K., Machine Interpretation of Line Drawings, MIT Press, Cambridge, Massachusets, London, England, 1986.

[White 86] White, N. Theory of Matroids, Encyclopedia of mathematics and its application, Cambridge University Press, Cambridge, London, New York, New Rochelle, Melboume, Sydney, 1986. 